\title{
Many-body coherent destruction of tunneling in photonic lattices
}

\author{
Stefano Longhi \\ Dipartimento di Fisica, Politecnico di Milano, Piazza L. da Vinci 32, I-20133 Milano, Italy
}

\begin{abstract}
An optical realization of the phenomenon of many-body coherent destruction of tunneling, recently predicted for interacting many-boson systems by Gong, Molina and Hänggi [Phys. Rev. Lett. 103, 133002 (2009)], is proposed for light transport in engineered waveguide arrays. The optical system enables a direct visualization in Fock space of the many-body tunneling control process.
\end{abstract}

PACS numbers: 03.65.Xp, 42.82.Et, 32.80.Qk

The phenomena of coherent destruction of tunneling (CDT) 1] and dynamic localization (DL) 2 represent seminal results in studies of quantum dynamical control. CDT and DL have been actively studied in several fields of physics [3], culminating in recent experiments on strongly driven Bose-Einstein condensates in optical lattices [4, 5] which provided a very direct observation of single-particle CDT and DL for matter waves. Many-body generalizations of CDT have attracted considerable interest in recent years, and experimentally [611. CDT for many interacting particles in a double well and its interplay with self trapping has been discussed in 6, whereas shaking-induced renormalization of the tunneling parameter and transition between superfluid and insulator states was predicted and observed in Refs. [7, 11. In Ref. 9], Gong, Molina and Hänggi recently proposed a novel route to CDT by considering a monochromatic fast modulation of the self-interaction strength of a Bose-Einstein condensate in a double well potential in the framework of a two-site Bose-Hubbard model. Very interestingly, the modulation can be tuned such that only an arbitrarily, a priori prescribed number of particles are allowed to tunnel. In another physical context, the idea of CDT was proposed [12, 13] and experimentally observed [14, 15] for light waves in coupled-waveguide structures, in which spatial propagation of light mimics the temporal dynamics of a a driven quantum particle in a bistable potential 16. The optical analogues studied in previous works [12 15] refer mostly to single-particle CDT. In the optical directional coupler proposed in Ref.[13], the addition of a Kerr nonlinearity enables to mimic CDT in presence of particle interaction in the mean-field limit, however such a scheme is not capable of simulating the two-mode Bose-Hubbard model. In a recent work [17, it was shown that a bosonic junction, originally realized using Bose-Einstein condensates in a double well potential [18, can be simulated using engineered waveguide lattices. In this Report it is shown that modulation of the optical lattice along the longitudinal direction enables to realize the phenomenon of many-body CDT in a purely classical setting. As compared to quantum simulators of the Bose-Hubbard dynamics, in which the measurable quantities are usually the 'macroscopic' boson number and phase difference in the two wells 18, the proposed optical setting offers the rather unique possibility of a direct visualization of the full many-body tunneling dy- namics in Fock space. Moreover, it offers the ability to prepare and observe small systems of particle numbers, allowing to study the transition between few-body and many-body behavior.

The starting point of our analysis is provided by a standard two-mode Bose-Hubbard Hamiltonian, which describes the tunneling dynamics of bosons in a doublewell potential (see, for instance, [9, 18])

$$
\hat{H}(t)=\frac{\hbar v}{2}\left(\hat{a}_{l}^{\dagger} \hat{a}_{r}+\hat{a}_{r}^{\dagger} \hat{a}_{l}\right)+\frac{\hbar g(t)}{4}\left(\hat{a}_{l}^{\dagger} \hat{a}_{l}-\hat{a}_{r}^{\dagger} \hat{a}_{r}\right)^{2},
$$

where $r$ (right) and $l$ (left) are the well sites, $\hat{a}_{k}$ and $\hat{a}_{k}^{\dagger}$ $(k=l, r)$ are the bosonic annihilation and creation operators, $v$ describes the constant tunneling rate between the two modes, and $g(t)$ is the modulated interaction strength between same-mode bosons. The total number of bosons $N=\hat{a}_{l}^{\dagger} \hat{a}_{l}+\hat{a}_{r}^{\dagger} \hat{a}_{r}$ is a conserved quantity and the dimension of the Hilbert space is $(N+1)$. A sinusoidal modulation $g(t)=g_{1} \sin (\omega t)$, without dc bias, is assumed for the interaction strength [19. To study the phenomenon of many-body CDT, in Ref. 9 ] a Floquet analysis of the Hamiltonian $\hat{H}(t)$ was performed after introduction of the Schwinger representation of the angular momentum operators. To provide an optical realization of the two-mode Bose-Hubbard Hamiltonian and to understand the many-particle CDT control scheme in an optical tunneling setup, we consider here the Fock space representation of the Hamiltonian $\hat{H}(t)$. If we expand the vector state of the system $|\psi(t)\rangle$ on the basis of Fock states with constant particle number $N$, i.e. after setting

$$
|\psi(t)\rangle=\sum_{l=0}^{N} \frac{c_{l}(t)}{\sqrt{l !(N-l) !}} \hat{a}_{1}^{\dagger l} \hat{a}_{2}^{\dagger N-l}|0\rangle
$$

the evolution equations for the amplitude probabilities $c_{l}(t)$ to find $l$ bosons in the left well and the other $(N-l)$ bosons in the right well, as obtained from the Schrödinger equation $i \hbar \partial_{t}|\psi(t)\rangle=\hat{H}|\psi(t)\rangle$, read explicitly [20]

$$
i \frac{d c_{l}}{d t}=\left(\kappa_{l} c_{l+1}+\kappa_{l-1} c_{l-1}\right)+g(t) V_{l} c_{l}
$$

$(l=0,1,2, \ldots, N)$, where we have set

$$
\kappa_{l}=\frac{v}{2} \sqrt{(l+1)(N-l)}, V_{l}=\frac{1}{4}(2 l-N)^{2} .
$$


The normalization condition $\sum_{l=0}^{N}\left|c_{l}(t)\right|^{2}=1$ holds. The evolution equations (3) for the Fock state amplitudes $c_{l}$ can be viewed as formally analogous to the coupled-mode equations describing light transport in a tight-binding array composed by $(N+1)$ waveguides with longitudinally-modulated propagation constant shift $g(t) V_{l}$ and engineered coupling rates $\kappa_{l}$ between adjacent waveguides, in which the temporal evolution of the Fock-state amplitudes of the Bose-Hubbard Hamiltonian is mapped into the spatial evolution of the modal field amplitudes of light waves in the various waveguides along the axial direction $t$ [17]. The fractional light power distribution $\left|c_{l}\right|^{2}$ in the various waveguides of the array at the propagation distance $t$ thus reproduces the distribution of occupation probabilities of the bosons between the two wells of the bistable potential. It is worth noticing that, in the absence of particle interaction, i.e. for $g=0$, the lattice model (3) associated to the Bose-Hubbard Hamiltonian was previously introduced in the photonic context to realize exact spatial beam self-imaging in finite waveguide arrays 21] and shown to belong to a rather general class of exactly-solvable self-imaging tight-binding lattices with equally-spaced energy levels 22. In the high-frequency modulation regime, the many-body CDT control method suggested in Ref. 9] can be simply explained as a tunneling inhibition process between certain waveguides in the array. After introduction of the slowly-varying amplitudes $a_{l}=c_{l} \exp \left[-i \int_{0}^{t} d \xi g(\xi)\right.$, application of the averaging method [23] yields the following evolution equations for the amplitudes $a_{l}$

$$
i \frac{d a_{l}}{d t}=\left(\sigma_{l} a_{l+1}+\sigma_{l-1}^{*} a_{l-1}\right)
$$

where we have set

$$
\sigma_{l}=\kappa_{l}\left\langle\exp \left[\left(V_{l}-V_{l-1}\right) \int_{0}^{t} d \xi g(\xi)\right]\right\rangle
$$

and the bracket stands for the time average over one oscillation cycle. Equations (5) and (6) provide a firstorder approximation to the exact tunneling dynamics in the lattice (for more details see [23]). For a sinusoidal modulation $g(t)=g_{1} \sin (\omega t)$ and using Eq.(4), the explicit form of the effective coupling rate $\sigma_{l}$ reads

$$
\sigma_{l}=\kappa_{l} \exp \left(i \varphi_{l}\right) J_{0}\left(\frac{g_{1}(N-2 l-1)}{\omega}\right)
$$

where $J_{0}$ is the Bessel function of order zero and $\varphi_{l}=$ $\left(g_{1} / \omega\right)(2 l-N-1)-l \pi / 2$ is an inessential phase term. Suppose now that the system is initially prepared with all the $N$ bosons on the right well, i.e. $c_{l}=\delta_{l, 0}$. According to Ref. [9], a desired number $l_{0}=0,1,2, \ldots$ of particles can be allowed to tunnel to the left well provided that the amplitude $g_{1}$ of the modulation is chosen in such a way that $g_{1}\left(N-2 l_{0}-1\right) / \omega$ is a root of the $J_{0}$ Bessel function. In this case, since the effective hopping rate $\sigma_{l_{0}}$ between the sites $l_{0}$ and $l_{0}+1$ vanishes, one has
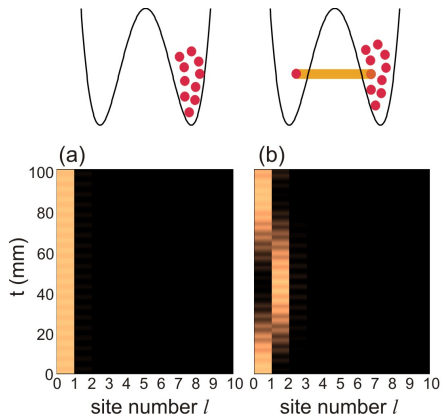

site number $l$
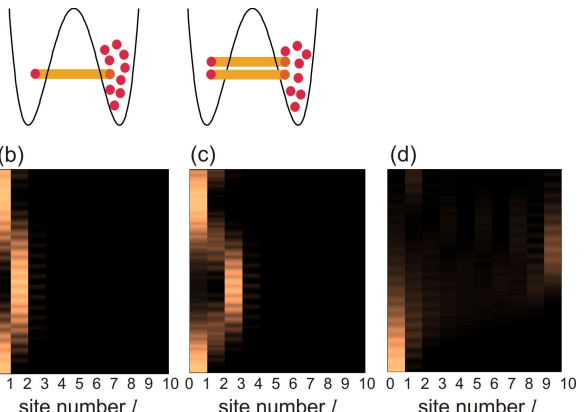

FIG. 1. (Color online) Evolution of the occupation probabilities $\left|c_{l}(t)\right|^{2}$ of $N=10$ bosons for the two-site Bose-Hubbard Hamiltonian (1) for $v=0.08 \mathrm{~mm}^{-1}, \omega=0.628 \mathrm{~mm}^{-1}$ and for a few values of the modulation amplitude $g_{1}$ : (a) $g_{1}=1.679 \times 10^{-4} \mathrm{~mm}^{-1} ;$ (b) $g_{1}=2.159 \times 10^{-4} \mathrm{~mm}^{-1}$, (c) $g_{1}=3.022 \times 10^{-4} \mathrm{~mm}^{-1}$, and (d) $g_{1}=1.346 \times 10^{-4} \mathrm{~mm}^{-1}$. The four values of the modulation amplitude $g_{1}$ correspond to the four vertical lines I-IV in the quasi-energy diagram of Fig.2(b). The modulation amplitudes in (a), (b) and (c) correspond to selective tunneling of zero, one and two bosons, respectively, as schematically indicated in the panels at the top of the figures.
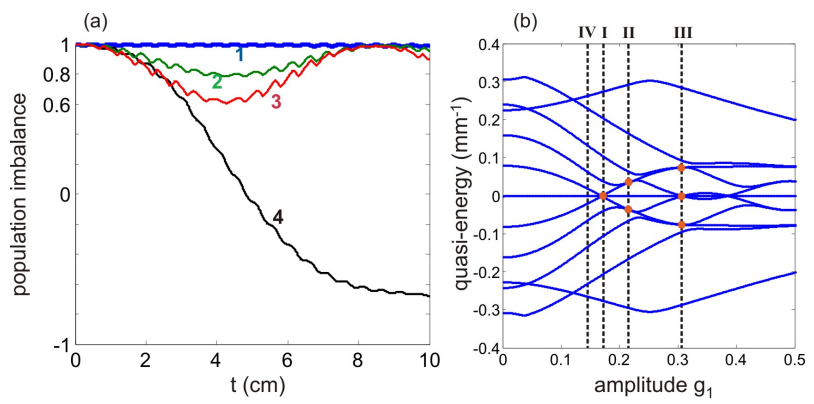

FIG. 2. (Color online) (a) Evolution of the normalized population imbalance $S(t)$ corresponding to the simulations shown in Fig. 1 (curve 1: $g_{1}=1.679 \times 10^{-4} \mathrm{~mm}^{-1}$; curve 2: $g_{1}=2.159 \times 10^{-4} \mathrm{~mm}^{-1}$; curve $3: g_{1}=3.022 \times 10^{-4} \mathrm{~mm}^{-1}$; curve 4: $g_{1}=1.346 \times 10^{-4} \mathrm{~mm}^{-1}$ ). (b) Quasi-energy spectrum of the two-site Bose-Hubbard Hamiltonian (1) versus $g_{1}$ for $v=0.08 \mathrm{~mm}^{-1}$ and $\omega=0.628 \mathrm{~mm}^{-1}$. The vertical lines I, II and III correspond to selective tunneling of zero, one and two bosons, respectively, as shown in Figs.1(a), (b) and (c).

$c_{l}(t)=0$ for $l \geq l_{0}+1$, and tunneling to the lattice sites $l=l_{0}+1, l_{0}+2, \ldots, N$ is thus forbidden. Since $\left|c_{l}(t)\right|^{2}$ is the probability to find $l$ bosons in the left well and the other $(N-l)$ bosons in the right well, this means that no more than $l_{0}$ particles can tunnel from the right to the left well. As discussed in Ref. 9], the conditions for selective CDT correspond to the crossing or touching of quasi-energy Floquet states of Eq.(3) with opposite parity. In our optical realization of the driven Bose-Hubbard Hamiltonian, the onset of many-body CDT is thus simply visualized as the inhibition of light tunneling in a portion of the array. As an example, Figs.1(a-c) show the evolution of the occupation probabilities $\left|c_{l}(t)\right|^{2}$, numerically computed from Eq.(3), corresponding to tun- 
neling of zero, one and two particles for a $N=10$ bosonic system, initially prepared with all the particles in the right well, for parameter values $v=0.08 \mathrm{~mm}^{-1}$, $\omega=0.628 \mathrm{~mm}^{-1}$ and for the three different values $g_{1}$ of the modulation satisfying the selective CDT conditions $g_{1}\left(N-2 l_{0}-1\right) / \omega=2.405$ with $l_{0}=0,1$ and 2 . The corresponding evolution of the normalized population imbalance [9], given by $S(t)=\sum_{l=0}^{N}[(N-2 l) / N]\left|c_{l}(t)\right|^{2}$, is depicted in Fig.2(a). The selective CDT realized in the three cases corresponds to the three points I, II and III in the quasi-energy diagram shown in Fig.2(b). For comparison, in Fig.1(d) and curve 4 of Fig.2(a) the behavior of the occupation probabilities and normalized population imbalance is also shown for a wrong value of the modulation amplitude $g_{1}$, which does not correspond to degeneracy of Floquet states [see line IV in Fig.2(b)]. Note that, as opposed to Figs.1(a)-(c), in this case the light waves spread over the entire waveguides of the array.

To realize the lattice model (3), the hopping rates and site potentials should be tailored according to Eqs.(4). In a waveguide array, this requires a control of waveguide spacing and of refractive index contrasts and/or channel sizes of the various waveguides. The tight-binding model (3) can be derived using a variational procedure starting from the paraxial and scalar wave equation for the electric field amplitude $\phi(x, t)$ describing the propagation of monochromatic light waves at wavelength $\lambda$ in an array of $(N+1)$ waveguides with a longitudinally-modulated refractive index profile $n(x, t)$ and substrate index $n_{s}$

$$
i \rtimes \partial_{t} \phi=-\frac{\lambda^{2}}{2 n_{s}} \partial_{x}^{2} \phi+\left[n_{s}-n(x, t)\right] \phi,
$$

where $\lambda=\lambda /(2 \pi)$ is the reduced wavelength of photons and $t$ is the longitudinal spatial coordinate. The hopping rates $k_{l}$ and propagation constant shifts $g V_{l}$ entering in the reduced equations (3) are given by certain overlapping integrals involving the modal field and index profiles of the guides (for details see, for instance, 24]). In particular, to independently engineer the coupling constants $\kappa_{l}$ and propagation constant shifts $g V_{l}$ of the waveguides, one can assume a chain of waveguides with equal normalized refractive index profile $G(x)$, but with different and longitudinallymodulated index contrasts $\Delta n_{l}(t) \quad(l=0,1,2, \ldots, N)$ and spacing $d_{l}=x_{l}-x_{l-1}(l=1,2, \ldots, N)$, i.e. $n(x, t)-n_{s}=\sum_{l=0}^{N} \Delta n_{l}(t) G\left(x-x_{l}\right)$. To realize the twomode Bose-Hubbard lattice (3), the values of $d_{l}$ and $\Delta n_{l}$ slightly vary around some mean values $d_{r}$ and $\Delta n$ that define a uniform array [17. The waveguide separation $d_{l}$ mainly determines the value of the coupling rate $\kappa_{l-1}$, with a characteristic exponential dependence of $\kappa_{l-1}$ from $d_{l}$, whereas the index change $\Delta n_{l}$ mainly defines the propagation constant mismatch $g_{1} V_{l}$. Numerical simulations were performed to reproduce the parameter conditions of Fig.1 for an operational wavelength $\lambda=633$ and assuming $n_{s}=1.45$, which applies to fused silica waveguide arrays. The normalized waveguide profile used in the simulations is given by $G(x)=$ (a)
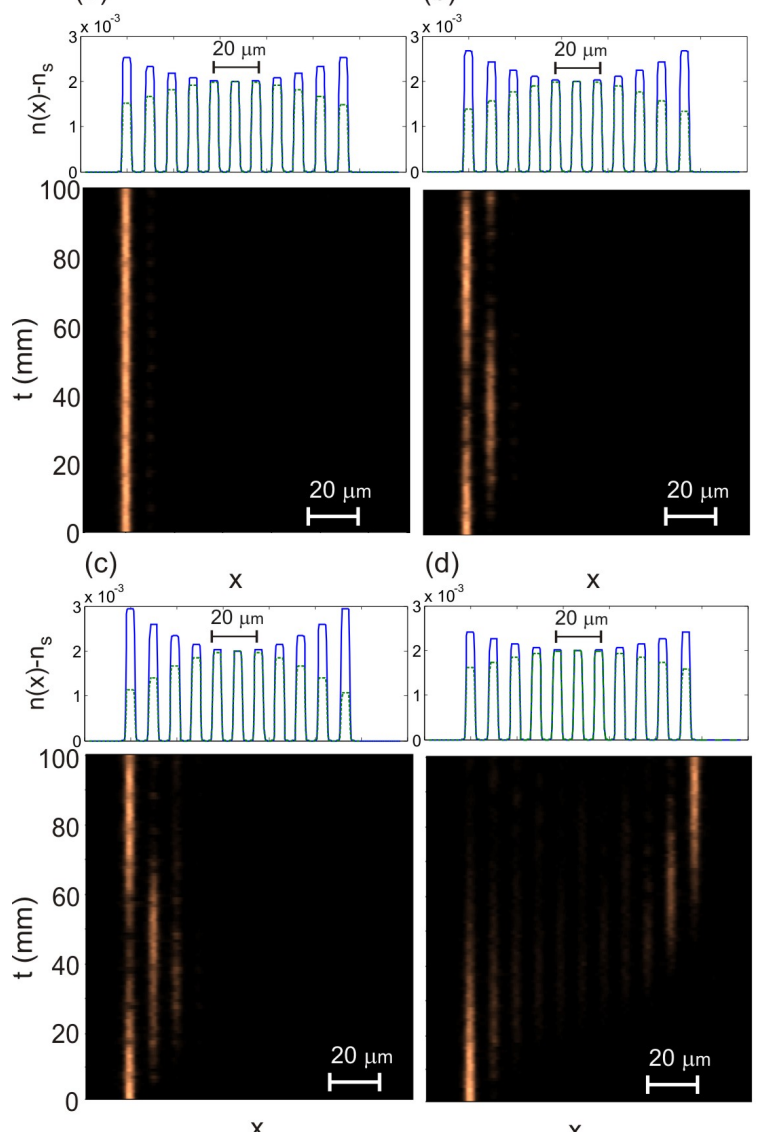

(d) $\quad x$
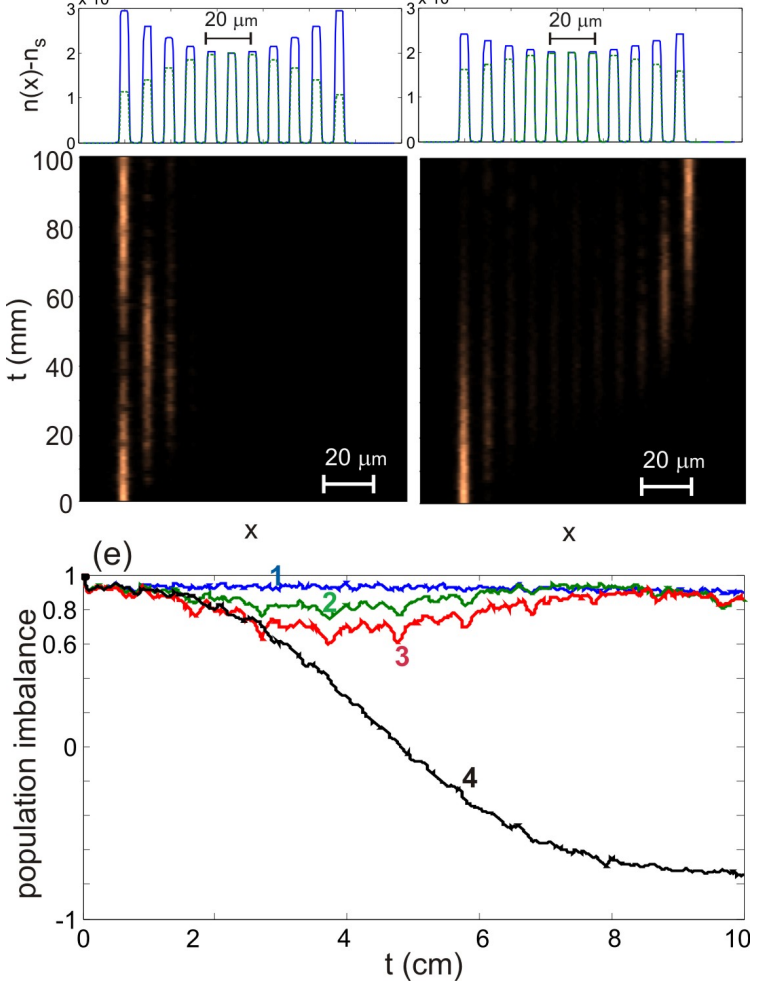

FIG. 3. (Color online) (a-d) Evolution of beam intensity (snapshot of $|\phi(x, t)|^{2}$ ) in four 10-cm-long longitudinallymodulated waveguide arrays, composed by $(N+1)=11$ waveguides, that realize the lattice models of Figs.1(a-d). The refractive index profiles $n(x)-n_{s}$ of the arrays, corresponding to the maximum/minimum of modulation amplitude $[\sin (\omega z)= \pm 1]$ are shown in the upper panels of the figures by the solid/dashed curves. The behavior of the normalized population imbalance $S(t)$, retrieved from the beam center of mass position, is shown in (e) for the four arrays.

$\left\{\operatorname{erf}\left[(x+w) / D_{x}\right]-\operatorname{erf}\left[(x-w) / D_{x}\right]\right\} /\left[2 \operatorname{erf}\left(w / D_{x}\right)\right]$, with channel width $2 w=4 \mu \mathrm{m}$ and diffusion length $D_{x}=0.3 \mu \mathrm{m}^{-1}$. To determine the distribution of distances $d_{l}$, a reference value $\Delta n=2 \times 10^{-3}$ of refractive index contrast was assumed, and correspondingly the coupling rate $\kappa$ between two adjacent 
waveguides versus distance $d$ was computed, yielding to a good approximation the exponential dependence $\kappa(d)=\kappa_{0} \exp \left[-\gamma\left(d-d_{r}\right)\right]$ for distances not too far from the reference distance $d_{r}=9 \mu \mathrm{m}$, where $\kappa_{0} \simeq 0.2144 \mathrm{~mm}^{-1}$ and $\gamma \simeq 0.6 \mu \mathrm{m}^{-1}$. The resulting distance distribution that realizes the coupling rates (4) with $v=0.08 \mathrm{~mm}^{-1}$ indicates that $d_{l}$ varies in the range 9-9.916 $\mu \mathrm{m}$. By modulating the index contrasts $\Delta n_{l}$ of the waveguides around the reference value $\Delta n$, four different arrays were then designed to realize the four different interaction regimes of Fig.1. A numerical computation of the propagation constant of the channel waveguide indicates that a propagation constant shift $g_{1} \sin (\omega t) V_{l}$ is approximately obtained by assuming a refractive index contrast $\Delta n_{l}=\Delta n+\beta g_{1} \sin (\omega t) V_{l} \lambda$, where $\Delta n=2 \times 10^{-3}$ is the reference value of the index contrast and $\beta$ is a numerical factor of order one $(\beta \simeq 1.23$ for the chosen error function waveguide profile). The resulting refractive index profile of the arrays, corresponding to the maximum $[\sin (\omega t)=1]$ and minimum $[\sin (\omega t)=-1]$ of the modulation cycle, are depicted in the upper panels of Figs.3(a-d). Such arrays could be fabricated in fused silica by the femtosecond laser writing technique 25, in which the different refractive index contrasts are obtained by varying the speed of the writing laser beam. Figures 3(a-d) show the evolution of light intensity $|\phi(x, t)|^{2}$ along the four arrays, as obtained by numerical simulations of Eq.(8) using a standard pseudospectral split-step method, when the left boundary waveguide is excited at the input plane. The behavior of the normalized population imbalance $S(t)$, which provides a clear signature of selective CDT [9], can be retrieved from a measurement of the beam center of mass position $\langle x(t)\rangle=\int d x x|\phi(x, t)|^{2} / \int d x|\phi(x, t)|^{2}$ using the simple relation $S(t) \simeq 1-2\langle x(t)\rangle /\left(N d_{r}\right)$ [17]. The corresponding behavior of $S(t)$ for the four arrays is depicted in Fig.3(e), which clearly indicates the onset of selective CDT according to the results shown in Figs.1 and 2 .

In conclusion, a classic wave optics realization of the selective many-body coherent destruction of tunneling, recently predicted for interacting many-boson systems in Ref. 9], has been proposed for light transport in modulated waveguide lattices. In such photonic structures light propagation in the various waveguides maps the evolution of the bosonic distribution in the double-well potential, and thus enables to visualize the entire many-body tunneling dynamics in the Fock space, a possibility which would be hard to achieve in matter wave systems.

This work was supported by the Italian MIUR (Grant No. PRIN-20082YCAAK, "Analogie ottico-quantistiche in strutture fotoniche a guida d'onda").
[1] F. Grossmann, T. Dittrich, P. Jung, and P. Hänggi, Phys. Rev. Lett. 67, 516 (1991); F. Grossmann, P. Jung, T. Dittrich, and P. Hänggi, Z. Phys. B: Condens. Matter 84, 315 (1991).

[2] D.H. Dunlap and V.M. Kenkre, Phys. Rev. B 34, 3625 (1986).

[3] M. Grifoni and P. Hänggi, Phys. Rep. 304, 229 (1998); S. Kohler, J. Lehmann, and P. Hänggi, Phys. Rep. 406, 379 (2005).

[4] H. Lignier, C. Sias, D. Ciampini, Y. Singh, A. Zenesini, O. Morsch, and E. Arimondo, Phys. Rev. Lett. 99, 220403 (2007).

[5] E. Kierig, U. Schnorrberger, A. Schietinger, J. Tomkovic, and M.K. Oberthaler, Phys. Rev. Lett. 100, 190405 (2008).

[6] M. Holthaus and S. Stenholm, Eur. Phys. J. B 20, 451 (2001).

[7] A. Eckardt, C. Weiss, and M. Holthaus, Phys. Rev. Lett. 95, 260404 (2005); A. Eckardt and M. Holthaus, Phys. Rev. Lett. 101, 245302 (2008).

[8] A. Eckardt and M. Holthaus, Europhys. Lett. 80 , 50004 (2007).

[9] J. Gong, L. Morales-Molina, and P. Hänggi, Phys. Rev. Lett. 103, 133002 (2009).

[10] C. Sias, H. Lignier, Y.P. Singh, A. Zenesini, D. Ciampini, O. Morsch, and E. Arimondo, Phys. Rev. Lett. 100, 040404 (2008).

[11] A. Zenesini, H. Lignier, D. Ciampini, O. Morsch, and E. Arimondo, Phys. Rev. Lett. 102, 100403 (2009).

[12] S. Longhi, Phys. Rev. A 71, 065801 (2005).
[13] X. Luo, Q. Xie, and B. Wu, Phys. Rev. A 76, 051802(R) (2007).

[14] G. Della Valle, M. Ornigotti, E. Cianci, V. Foglietti, P. Laporta, and S. Longhi, Phys. Rev. Lett. 98, 263601 (2007).

[15] A. Szameit, Y. V. Kartashov, F. Dreisow, M. Heinrich, T. Pertsch, S. Nolte, A. Tünnermann, V. A. Vysloukh, F. Lederer, and L. Torner, Phys. Rev. Lett. 102, 153901 (2009); A. Szameit, Y. V. Kartashov, M. Heinrich, F. Dreisow, R. Keil, S. Nolte, A. Tnnermann, V. A. Vysloukh, F. Lederer, and L. Torner, Opt. Lett. 34, 2700 (2009).

[16] S. Longhi, Laser Photon. Rev. 3, 243 (2009).

[17] S. Longhi, J. Phys. B 44, 051001 (2011).

[18] M. Albiez, R. Gati, J. Fölling, S. Hunsmann, M. Cristiani, and M.K. Oberthaler, Phys. Rev. Lett. 95, 010402 (2005).

[19] As discussed in Ref. 9], a dc bias of $g(t)$ can induce population localization via a self-trapping mechanism. To isolate population localization due to possible CDT phenomena from that due to self-trapping, we will consider here solely an ac modulation.

[20] V.S. Shchesnovich and M. Trippenbach, Phys. Rev. A 78, 023611 (2008).

[21] R. Gordon, Opt. Lett. 29, 2752 (2004).

[22] S. Longhi, Phys. Rev. B 82, 041106 (2010).

[23] S. Longhi, Phys. Rev. B 77, 195326 (2008).

[24] S. Longhi, Phys. Lett. A 359, 166 (2006).

[25] A. Szameit and S. Nolte, J. Phys. B 43, 163001 (2010). 